DOI: $10.34185 / 1991-7848.2019 .01 .11$

УДК 669.187

С.Н. Тимошенко, М.В. Губинский

\title{
ЭНЕРГОЭФФЕКТИВНЫЕ РЕШЕНИЯ ДИАМЕТРА РАСПАДА ЭЛЕКТРОДОВ ДУГОВОЙ СТАЛЕПЛАВИЛЬНОЙ ПЕЧИ
}

В традиционных решениях дуговых сталеплавильных печей (ДСП) диаметр распада электродов минимизируют для снижения излучения открытых электрических дуг на стены в период доводки стали. В связи с этим возникают негативные эффекты связанные с неравномерностью тепловыделения в объеме шихты в период плавления, которую в определенной мере компенсируют топливно-кислородные горелки. В ДСП высокой мощности $c$ интенсивной технологией $u$ широким применением водоохлаждаемых панелей, актуальна оптимизация диаметр распада электродов по критерию максимальной относительной продолжительности закрытого горения дуг в проплавляемых в шихте колодцах, как энергоэффективного этапа наиболее энергоемкого периода плавления. Целью работы является повышение энергоэффективности ДСП путем оптимизации диаметра распада электродов. Методика исследований - численное моделирование процессов нагрева и плавления скрапа энергией дугового разряда. Численное моделирование теплообмена в рабочем пространстве ДСП показало, что для печей с интенсивной технологии увеличения диаметра распада электродов из традиционного 0,2 до 0,42-0,49 внутреннего диаметра каркаса повышает энергоэффективность ДСП за счет роста относительного времени закрытого горения дуг. Изменение режима плавления приводит к экономии электроэнергии на 5-6\% в мощных печах (120-250 m) и на 2-3\% в печах малой вместимости (12-15 m).

Ключевые слова: дуговая сталеплавильная печь, теплообмен в период плавления, диаметр распада электродов, энергоэффективность.

\section{Постановка проблемы}

В традиционных решениях дуговых сталеплавильных печей (ДСП) диаметр распада электродов $D_{\mathrm{P}}$ минимизируют для снижения излучения открытых электрических дуг на стены в период доводки стали [1]. Недостатком является неравномерность тепловыделения в объеме шихты в период плавления, которую в определенной мере компенсируют топливно-кислородные горелки. В ДСП высокой мощности с интенсивной технологией и широким применением водоохлаждаемых панелей, актуальна оптимизация $D_{\mathrm{p}}$ по критерию максимальной относительной продолжительности закрытого горения дуг в проплавляемых в шихте колодцах, как энергоэффективного этапа наиболее энергоемкого периода плавления [2].

\section{Анализ последних исследований и публикаций}

Численные модели V. Logar, D. Dovžan, I. Škrjanc [3]; F.Opitz и P. Treffinger [4], a также Ю. Станкевич и др. [5] с определенными допущениями описывают динамику плавления однородной шихты энергией излучения дугового разряда.

(c) Тимошенко С.Н., Кубинский М.В., 2019 
В рамках сопряженной задачи переноса теплоты и массы решается система уравнений сохранения массы, импульса и энергии в средах с учетом движения границ сред и фазовых переходов, что позволяет, в частности, дать оценку влияния насыпной плотности скрапа $\rho_{\text {н }}$ на динамику его плавления. Для 100-т ДСП продолжительность плавления снижается примерно вдвое на шредерном скрапе $\left(\rho_{\mathrm{H}}=0,8 \mathrm{~T} / \mathrm{M}^{3}\right)$ в сравнении с оборотным $\left(\rho_{\mathrm{H}}=1,8 \mathrm{~T} / \mathrm{M}^{3}\right)$ [4], что с учетом влияния $\rho_{\mathrm{H}}$ на угар железа [6], позволяет прогнозировать технико-экономические показатели печи. По расчетному перемещению образующей стенки колодца производится оценка потерь теплоты через водоохлаждаемые поверхности [3], которая согласуется с практикой. Вместе с тем, вопросы оптимизации $D_{\mathrm{p}}$ в ДСП в литературе практически не отражены.

\section{Цель и методика исследований}

Целью работы является повышение энергоэффективности ДСП путем оптимизации диаметра распада электродов. Методика исследований - численное моделирование процессов нагрева и плавления скрапа энергией дугового разряда.

\section{Основной материал исследований}

Схема, отражающая геометрическую постановку задачи, приведена на рис. 1.

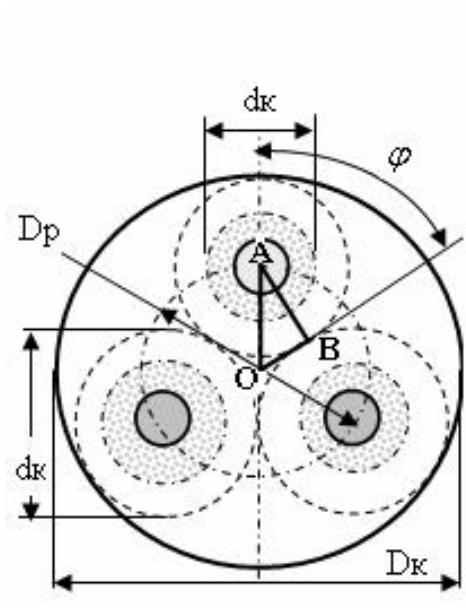

a

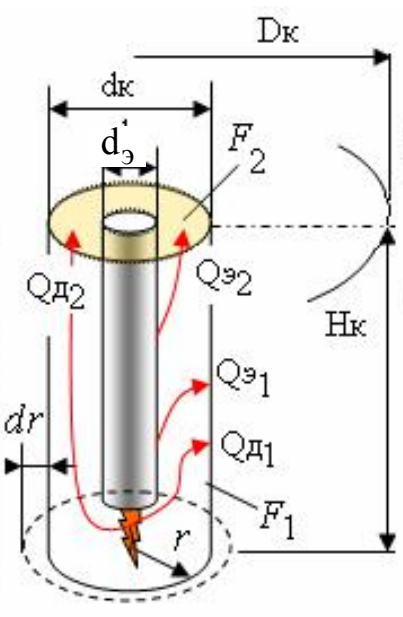

6

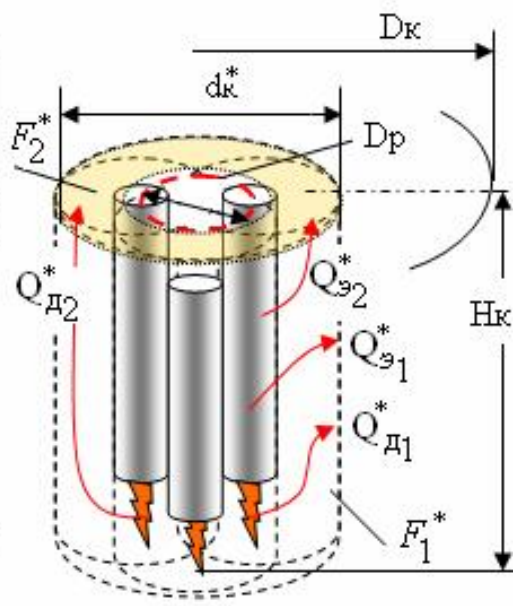

$\mathrm{B}$

Рисунок 1 - Схема плавления шихты в геометрической постановке (а). Теплообмен в индивидуальных (б) и общем (в) колодцах. Обозначения - в тексте

Геометрическая постановка задачи. Из треугольника ОАВ с углом $\varphi=60^{\circ}$ оптимальная величина $D_{\mathrm{P}}^{*}=0,54 D_{\mathrm{\kappa}}$ определяется при выполнении условия: $\left(D_{\mathrm{p}} / 2\right) \sin 60^{\circ}=\left(D_{\mathrm{K}} / 2\right)-\left(D_{\mathrm{p}} / 2\right)$. Она хорошо согласуется с известными решениями руднотермических печей, работающих в режиме закрытого горения дуг. Ввиду неоднозначности влияния $D_{\mathrm{p}}$ на энергоэффективность печи в периоды плавления и нагрева ванны, необходимо рассмотреть ее теплотехнологические аспекты. 
Теплотехнологическая постановка задачи. Приняты следующие допущения. Шихта - однородный шредерный скрап с насыпной плотностью $\rho_{\text {н }}$ загружается в печь в один прием. Электрическая мощность выделяется в трех дугах, горящих концентрично электродам, равномерно, что обеспечивает при опускании электродов равномерное плавление шихты с формированием концентричных колодцев с устойчивыми стенками. Диаметр и глубина колодца $d_{\mathrm{\kappa}}=1,35 \cdot d_{\ni}[4,5]$ и $H_{\mathrm{\kappa}}$ соответственно. Температура и степень черноты шихты и ВЭ свода 293 К и 1,0 соответственно [2].

Расчетная схема теплообмена излучением в индивидуальном и общем колодцах и параметры, характеризующие расширение колодцев и усвоение энергии шихтой, приведены на рис. 1б, в. Задача оптимизации $D_{\mathrm{P}}$ включает:

- оценку частного коэффициента энергоэффективности дуги при эволюции колодцев путем расчета мощности излучения, анализа ее полезного использования и потерь;

- оценку относительного времени закрытого (в колодце) и открытого горения дуг в общей продолжительности плавления, как функции $D_{\mathrm{P}}$;

- анализ энергоэффективности ДСП при варьировании $D_{\mathrm{P}}$.

Источники излучения в модели: дуга и поверхность электродов в колодцах. Приемники излучения: цилиндрическая поверхность стен $F_{1}, F_{1}^{*}$ колодца (полезная энергия) и кольцевая поверхность свода $F_{2}, F_{2}{ }^{*}$ над колодцем (потери теплоты с водой).

Потери теплоты через электроды в окружающую среду свободной конвекцией и излучением в энергобалансе не превышают в сумме 1 \% и в данной задаче игнорируются.

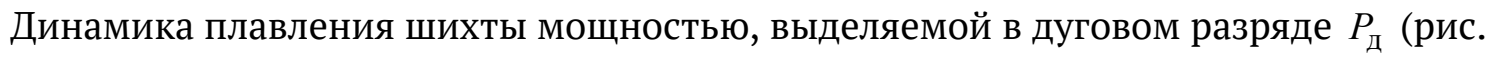
1б), представлена, как временная последовательность квазистационарных состояний кольцевых слоев скрапа толщиной $d r$ в эволюционирующем колодце текущего радиуса $r$. Энергетический баланс этапа $\tau_{\mathrm{i}}$ периода плавления имеет вид:

$$
P_{\text {д }} \eta_{\text {дк }} \int_{0}^{\tau_{\mathrm{i}}} d \tau=2 \pi H_{\mathrm{\kappa}} \rho_{\mathrm{H}} k_{\mathrm{p}}\left[\int_{A}^{B} \Delta t(r) \cdot C_{\mathrm{c}}(r) \cdot r d r+q_{\text {пл }} \int_{A}^{B} r d r\right] \cdot k_{\mathrm{B}}
$$

где $\eta_{\text {дк }}$ - частный коэффициент энергоэффективности дуги в колодце; $\Delta t(r), C(r)$ текущие перепад температуры в слое скрапа и теплоемкость скрапа соответственно;

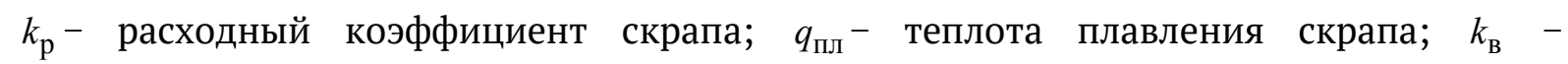
коэффициент увеличения энергозатарт на нагрев притока воздуха в печь; $A, B$ пределы интегрирования в соответствии с геометрией колодцев на данном этапе плавления.

Исследования выполнены для трех типоразмеров ДСП вместимостью 15, 120, 250 т с интенсивной технологией и загрузкой скрапа бадьями. 
Мощность излучения дуг и электродов в колодцах определяется по методике [7]. Результаты расчетов приведены на рис. 2а,6 в виде диаграмм, характеризующих соотношение полезной, усвоенной шихтой мощности излучения дуги и электрода $Q_{д 1}, Q_{\ni 1}$ в индивидуальных колодцах или системы дуг и электродов $Q_{\text {д1 }}^{*}, Q_{\ni 1}^{*}$ в общем колодце, и мощности потерь теплоты с водой в своде, выраженной для индивидуального и общего колодцев как $Q_{д 2}, Q_{\ni 2}$ и $Q_{д 2}^{*}, Q_{\ni 2}^{*}$ соответственно(см. рис. 1 ).

В индивидуальном и общем колодцах расчетные потери энергии излучения и частные коэффициенты энергоэффективности составляют $6 \%\left(\eta_{д к}=0,94\right)$ и $30 \%$ $\left(\eta_{\text {дк }}{ }^{2}=0,70\right)$ соответственно. Показатели относятся к системе дуга - электрод, в которой преобладает излучение дуги (80-85\%).

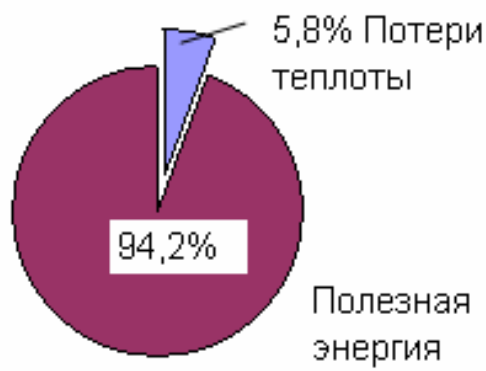

a

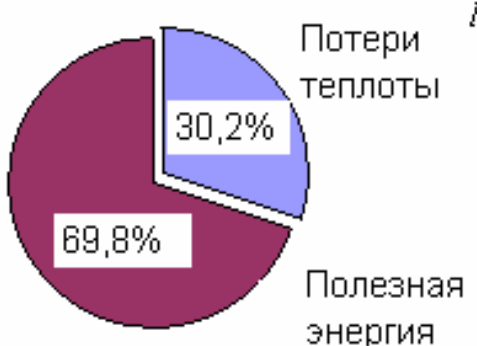

6

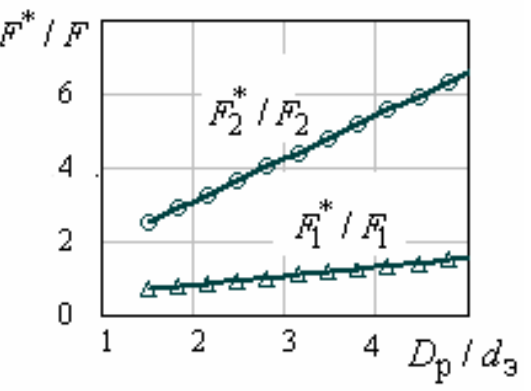

B

Рисунок 2 - Теплоэнергетические показатели процесса плавления шихты в индивидуальных колодцах (а) и общем колодце (б). Изменение геометрии колодцев при варьировании $D_{\mathrm{p}} / d_{\ni}($ в). Обозначения - в тексте и на рис. 1

На стадии плавления в индивидуальных колодцах, величина $\eta_{д к ~}$ в процессе их эволюции в среднем на $(94-70) / 2=12 \%$ выше, чем $\eta_{\text {дк }}^{*}$ в общем колодце. Результат объясняется опережающим ростом поверхности свода $F_{2}^{*} / F_{2}$ (сток теплоты с водой) относительно поверхности стен колодца $F_{1}^{*} / F_{1}$ (характеризует усвоение теплоты шихтой) при слиянии колодцев и с увеличением $D_{\mathrm{p}} / d_{\text {э }}$ (рис. 2в).

Основываясь на оценках $\eta_{\text {дк }}$, рассмотрим влияние $D_{\mathrm{p}}$ на относительную продолжительность плавления скрапа в колодцах, с учетом эволюции последних, с целью оптимизации $D_{\mathrm{p}} / D_{\text {к }}$ по критерию минимальных удельных энергозатрат.

Продолжительность формирования колодца $\tau_{\text {к0 }}$ определяется из частного энергетического баланса плавления скрапа массой $m_{\mathrm{K}}=\pi\left(d_{\mathrm{K}}{ }^{2} / 4\right) \rho_{\mathrm{H}} H_{\mathrm{K}}$ при расчетном $\eta_{\text {дк }}=0,94$. Принято допущение, что энергия дугового разряда не передается окружающей шихте с высокой порозностью и, соответственно, низкой эффективной

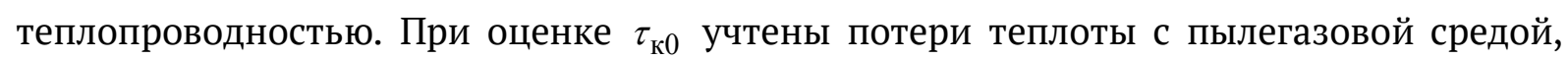
формируемой в период плавления преимущественно притоком воздуха $Q_{\mathrm{B}}=175$ $\mathrm{HM}^{3} / \mathrm{T}$, который нагревается до средней температуры $t_{\mathrm{B}}=900^{\circ} \mathrm{C}$ от исходной $t_{0}=20^{\circ} \mathrm{C}$. 


$$
\left.\tau_{\kappa 0}=\left[\left[m_{\mathrm{K}} k_{\mathrm{p}}\left(C_{\mathrm{c}} \Delta t_{\mathrm{c}}+q_{\text {пл }}\right)\right]+Q_{\mathrm{B}} \rho_{\mathrm{B}} C_{\mathrm{B}}\left(t_{\mathrm{B}}-t_{0}\right)\right] /\left[\left(P_{\mathrm{a}} k_{\mathrm{H}} / 3\right) \eta_{\text {дк }}\right)\right]
$$

где $\Delta t_{\mathrm{c}}-$ разность между температурой плавления скрапа $\left(1520^{\circ} \mathrm{C}\right)$ и температурой исходной шихты $\left(20^{\circ} \mathrm{C}\right) ; k_{\mathrm{H}}$ - коэффициент снижения электрической мощности в начале плавления $(0,85)[2] ; C_{\text {в }}$-средняя теплоемкость воздуха; $\rho_{\mathrm{B}}-$ плотность воздуха при н.у.

Для 120-т ДСП при $d_{\ni}=0,61 \mathrm{M}, H_{\mathrm{K}}=3,5$ м получено $\tau_{\text {к0 }}=139$ сек., что находится в соответствии с известными оценками $[4,5]$.

Из (2) определен коэффициент увеличения энергозатрат на нагрев приточного воздуха, используемый в дальнейших расчетах, $k_{\mathrm{B}}=1,16$.

Используя (1), выполнен расчет продолжительности этапов периода плавления шредерного скрапа с характерным размером фрагментов $b$ при эволюции колодцев. Исходные параметры, включая пределы интегрирования в (1) и, $\eta_{\text {дк }}$ приведены в табл. 1.

Таблица 1

Параметры расчета длительности плавления. Обозначения - в тексте и на рис. 2.

\begin{tabular}{|c|c|c|c|c|}
\hline \multirow[t]{2}{*}{ Этап периода плавления } & \multicolumn{2}{|c|}{$\begin{array}{l}\text { Пределы интегрирования } \\
\text { уравнении (1) }\end{array}$} & \multirow{2}{*}{$\begin{array}{l}\text { Коэффициент } \\
\text { энерго- } \\
\text { эффективно- } \\
\text { сти дуги }\end{array}$} & \multirow{2}{*}{$\begin{array}{l}\text { Мощ- } \\
\text { ность } \\
\text { дуги } \\
P_{\text {д }}\end{array}$} \\
\hline & Нижний А & Верхний В & & \\
\hline $\begin{array}{l}\tau_{\text {к1 }} \text {-время } \quad \text { расширения } \\
\text { колодцев до корпуса печи }\end{array}$ & $d_{\mathrm{K}} / 2$ & {$\left[\left(D_{\mathrm{K}}-D_{\mathrm{p}}\right) / 2\right]-\mathrm{b}$} & $\left(\eta_{\text {дК }}+\eta_{\text {дК }}^{*}\right) / 2$ & $P_{\mathrm{a}} / 3$ \\
\hline $\begin{array}{l}\tau_{\text {к11 }} \text {-время расширения } \\
\text { колодцев до слияния. }\end{array}$ & $d_{\mathrm{K}} / 2$ & $\left(D_{\mathrm{p}} / 2\right) \sin 60^{\circ}-\mathrm{b} / 4$ & $\left(\eta_{\text {дк }}+\eta_{\text {дк }}^{*}\right) / 2$ & $P_{\mathrm{a}} / 3$ \\
\hline $\begin{array}{l}\tau_{\text {к2 }} \text {-время расширения } \\
\text { общего колодца до корпуса } \\
\text { печи. }\end{array}$ & $\left(D_{\mathrm{p}}+d_{\mathrm{K}}\right) / 2$ & $\left(D_{\mathrm{K}} / 2\right)-\mathrm{b}$ & $\eta_{\text {дк }}^{*}$ & $P_{\mathrm{a}}$ \\
\hline
\end{tabular}

Приняты следующие допущения:

- текущий перепад температуры в слое скрапа $\Delta t(r)$ линейно зависит от радиальной координаты: в начальный момент $\Delta t\left(d_{\mathrm{K}} / 2\right)=\Delta t_{\mathrm{c}}-W_{\text {эк }} /\left(m_{\mathrm{K}} C_{\mathrm{c}}\right)$, что является следствием повышения средней температуры завалки массой $m=\pi\left(D_{\mathrm{K}}^{2} / 4\right) \rho_{\mathrm{H}} H_{\mathrm{\kappa}} \quad$ после ввода электроэнергии $W_{\text {эк }}=P_{\mathrm{a}} k_{\mathrm{H}} \tau_{\mathrm{\kappa} 0} \eta_{\text {дк }} /\left(k_{\mathrm{p}} k_{\mathrm{B}}\right)$ за время формировании колодцев. На конечном этапе плавления шихты $\Delta t\left(D_{\mathrm{K}}\right) \rightarrow 0$. Зависимость в итоге имеет вид:

$$
\Delta t(r)=\left[\left(D_{\mathrm{K}} / 2\right)-r\right] \cdot\left[\Delta t_{\mathrm{c}}-W_{\text {эК }} /\left(m_{\mathrm{K}} C_{\mathrm{c}} k_{\mathrm{B}}\right)\right] /\left[\left(D_{\mathrm{K}}-d_{\mathrm{K}}\right) / 2\right] ;
$$

- теплоемкость скрапа, будучи функцией температуры, является и функцией радиальной координаты $C_{\mathrm{c}}(r)$. На основе данных о $\Delta t(r)$, температуре плавления скрапа и $C_{\mathrm{c}}$ в интересующем интервале температур, в пакете «Excel» получена

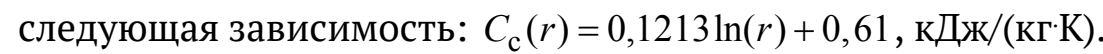


- расширение колодцев осуществляется до достижения остаточной толщины слоя шихты, равной половине характерного размера шредерного скрапа $b=0,5 \mathrm{M}$, после чего происходит обрушение стен колодца и их слияние. При выходе образующей колодца на расстояние $b$ до периметра печи, его функция завершается: при высокой порозности шихты полагаем, что излучаемая мощность теряется в вохоохлаждаемых панелях.

Общая продолжительность закрытого горения дуг при плавлении скрапа в колодцах $\tau_{\text {зд }}$ включает сумму составляющих из табл. 1, где из величин $\tau_{\text {к1 }} \tau_{\text {к11 }}$ принимается минимальное значение: $\tau_{\text {зд }}=\tau_{\mathrm{\kappa} 0}+\min \left(\tau_{\mathrm{\kappa} 1}, \tau_{\mathrm{\kappa} 11}\right)+\tau_{\mathrm{\kappa} 2}$.

Время плавления загруженной шихты $\tau_{\text {п }}$ рассчитывается из энергетического баланса с учетом стоков теплоты, включая приток воздуха в печь и шлакообразование, методом последовательных приближений. Оценочная величина $\eta_{д}$ в начальном приближении $\bar{\eta}_{\text {д1 }}=\left[\left(\eta_{\text {д }}+\eta_{\text {д }}{ }^{*}+\eta_{\text {од }}\right) / 3\right]$ принята, как среднее значение составляющих.

$$
\tau_{\text {П }}=\left[m k_{\mathrm{p}} k_{\mathrm{B}}\left(C_{\mathrm{c}} \Delta t_{\mathrm{c}}+q_{\text {пл }}\right)+k_{\text {шп }} m\left(C_{\text {ш }} \Delta t_{\text {ш }}+q_{\text {плш }}\right)\right] /\left(P_{\mathrm{a}} \bar{\eta}_{\text {д }}\right)
$$

где $C_{\text {ш }}$-средняя в интервале $\Delta t_{\text {ш }} \simeq \Delta t_{\mathrm{c}}$ теплоемкость шлакообразующих; $q_{\text {плш }}$ средняя теплота плавления шлакообразующих; $k_{\text {шп }}$ - кратность шлака.

Для оценки частного коэффициента энергоэффективности открытой дуги $\eta_{\text {од }}$ выполнен анализ использования активной электрической мощности $P_{\mathrm{a}}$ на нагрев расплава. Были учтены основные стоки энергии в ДСП: с водой $Q_{\mathrm{oв}}$ [7], и с пылегазовой средой $Q_{\text {пгс }}$ со средней температурой $1000{ }^{\circ} \mathrm{C}$. Начальное приближение длительности периода нагрева, согласно промышленным данным [2], принято 15 мин. Расчеты выполнены в пакете «Mathcad 14».

$$
\eta_{\text {од }}=\left[P_{\mathrm{a}}-\left(Q_{\mathrm{oв}}+Q_{\text {пгс }}\right)\right] / P_{\mathrm{a}}
$$

Для 120-т ДСП оценка $\eta_{\text {од }}=0,489$. В последующих итерациях в (3) при расчете $\tau_{\text {П }}$ использовано средневзвешенное значение частного коэффициента энергоэффективности дуги:

$$
\bar{\eta}_{\text {д }}=\eta_{\text {дк }} \cdot \frac{\tau_{\mathrm{\kappa} 0}}{\tau_{\Pi}}+\frac{\eta_{\text {дК }}+\eta_{\text {дК }}^{*}}{2} \cdot \frac{\min \left(\tau_{\mathrm{\kappa} 1}, \tau_{\mathrm{\kappa} 11}\right)}{\tau_{\Pi}}+\eta_{\text {дк }}^{*} \cdot \frac{\tau_{\mathrm{\kappa} 2}}{\tau_{\Pi}}+\frac{\eta_{\text {дК }}^{*}+\eta_{\text {од }}}{2} \cdot \frac{\tau_{\Pi}-\tau_{\kappa}}{\tau_{\Pi}}
$$

до получения устойчивого результата по $\tau_{\text {п }}$ (на третьей итерации).

Продолжительность открытого горения дуг $\tau_{\text {од }}$ после обрушения стен колодца определяется разностью времени плавления $\tau_{\text {п }}$ всей завалки массой $m=\pi\left(D_{\mathrm{\kappa}}^{2} / 4\right) \rho_{\mathrm{H}} H_{\mathrm{\kappa}} k_{\mathrm{p}}$ и значением $\tau_{\text {зд }}$, т.е. $\tau_{\text {од }}=\tau_{\text {п }}-\tau_{\text {зд }} \cdot$

Результаты расчета относительного времени закрытого $\tau_{\text {зд }} / \tau_{\text {П }}$, открытого горения дуг $\tau_{\text {од }} / \tau_{\text {п }}$ и операций в индивидуальных $\tau_{\text {к1 }} / \tau_{\text {п }}$ и общем $\tau_{\text {к2 }} / \tau_{\text {п }}$ колодцах для 120-т ДСП при варьировании $D_{\mathrm{p}}$, выполненного в пакете «Mathcad 14», показаны на рис. 3а. 
С ростом $D_{\mathrm{p}} / D_{\text {к }}$ увеличивается относительная продолжительность работы дуг в индивидуальных колодцах $\tau_{\text {к1 }} / \tau_{\text {п }}$, достигая максимума при $D_{\mathrm{p}}^{\text {opt }}=0,46 D_{\text {к }} 27 \%$ времени плавления. Относительное время закрытого горения дуг в колодце $\tau_{\text {зд }} / \tau_{\text {п }}$ при $D_{\mathrm{p}}{ }^{\text {опт }}$ приближается к 52 \%, а относительная продолжительность работы в общем колодце $\tau_{\text {к2 }} / \tau_{\text {п }}$, как менее энергоэффективный режим, снижается с 36 до 20 \% времени плавления, тогда как относительная доля наиболее энергозатратного режима открытого горения дуг $\tau_{\text {од }} / \tau_{\text {п }}$ уменьшается с 59 до $48 \%$.

На рис. 36 представлены интегральные показатели, характеризующие энергоэффективность периода плавления при $D_{\mathrm{p}}$ опт относительно базовой величины $D_{\mathrm{p}}^{\sigma} \simeq 0,2 D_{\mathrm{\kappa}}$. Так, индекс продолжительности закрытого горения дуг $\beta_{3 д}=\tau_{\text {зд( }\left(D_{\mathrm{p}}^{\text {opt }}\right)} / \tau_{\text {зд( }\left(D_{\mathrm{p}}^{6}\right)}$ возрастает на $16 \%$, а индекс продолжительности открытого горения дуг $\beta_{\text {од }}$ и индекс продолжительности периода плавления $\beta_{\text {п }}$, определяемые аналогично $\beta_{3 д}$, снижаются на $15 \%$ и $4 \%$.
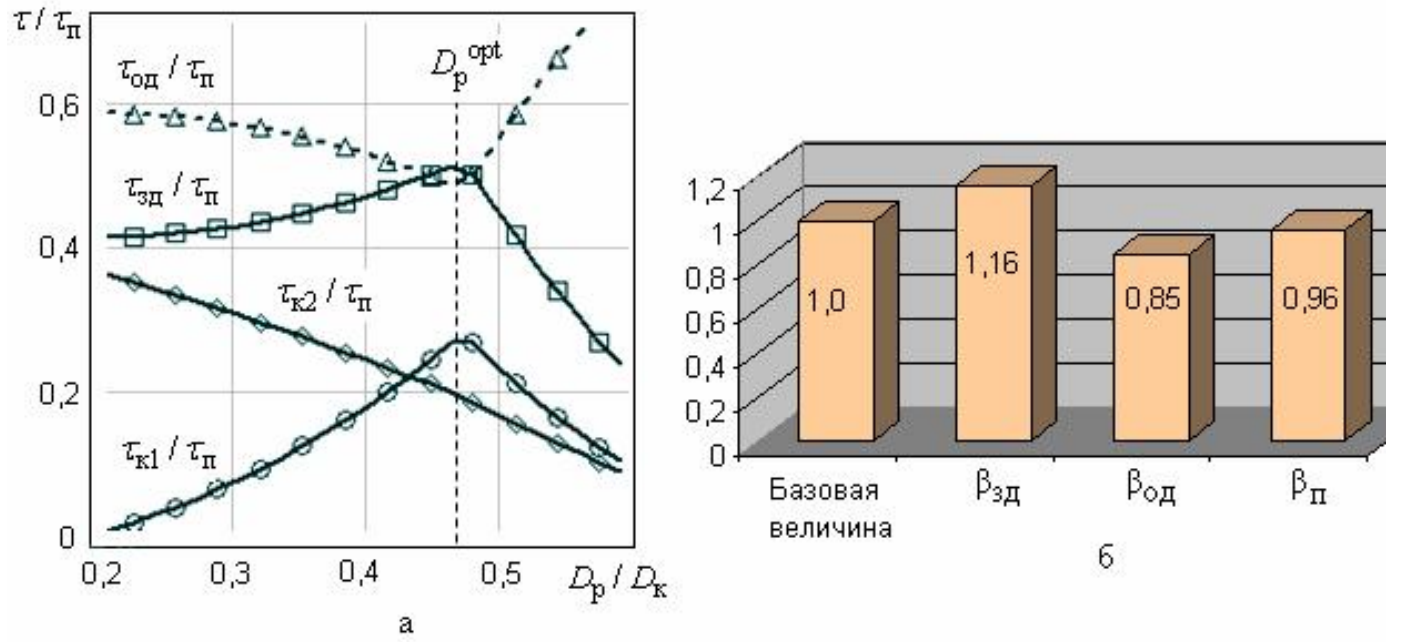

Рисунок 3 - Зависимость относительного времени $\tau / \tau_{\text {п }}$ горения дуг в колодцах и

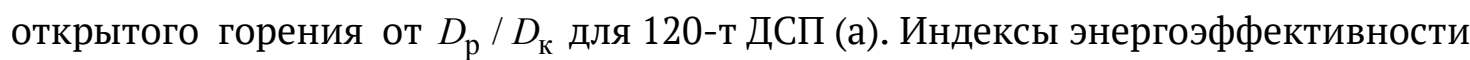
плавления при оптимальном $D_{\mathrm{p}}$ относительно базового (б). Обозначения - в тексте

Смещение оптимального значения в сторону уменьшения от геометрического решения связано с принятым допущением об остаточной толщине стен колодца, не экранирующей излучение дуг на водоохлаждаемые панели, и изменением частного коэффициента энергоэффективности дуги при эволюции колодца.

Исследование влияния $D_{\mathrm{p}}$ на режим плавления и удельный расход электроэнергии в наиболее энергоемкий период плавления выполнено для промышленных 15, 120 и 250-т ДСП на основании (2-4) в пакете «Mathcad 14». Абсолютное и относительное сокращение расхода электроэнергии при варьировании 
$D_{\mathrm{p}}$ составляет $\Delta W_{\text {эп }}=\left(P_{\mathrm{a}} / m\right)\left(\tau_{\Pi\left(D_{\mathrm{p}}^{6}\right)}-\tau_{\left.\text {п( } D_{\mathrm{p}}\right)}\right)$, кВтч/т $\quad$ и $\left.\quad \beta_{\text {эп }}=100 \Delta W_{\text {эп }} /\left[\left(P_{\mathrm{a}} / m\right) \tau_{\Pi\left(D_{\mathrm{p}}^{6}\right)}\right)\right], \%$. соответственно. Результаты исследования представлены на рис. 4.

Оптимальный $D_{\mathrm{p}}$ с ростом вместимости печи $\mathrm{M}$ увеличивается с $0,42 D_{\text {к }}$ ( $\mathrm{M}=15 \mathrm{~T})$ до $0,49 D_{\mathrm{K}}(\mathrm{M}=250 \mathrm{~T})$. Энергоэффективность ДСП определяется соотношением $\tau_{\text {зд }}$ и $\tau_{\text {од }}$ (рис. $\left.4 \mathrm{a}\right)$ и при оптимизации $D_{\mathrm{p}}$, повышается с ростом вместимости печи. Следствием изменения режима плавления является экономия электроэнергии (рис. $46)$, составляющая в крупных печах (120-250 т) 5-6\%, а в печах малой вместимости (12-15 т) до 2-3\% от среднего удельного расхода.

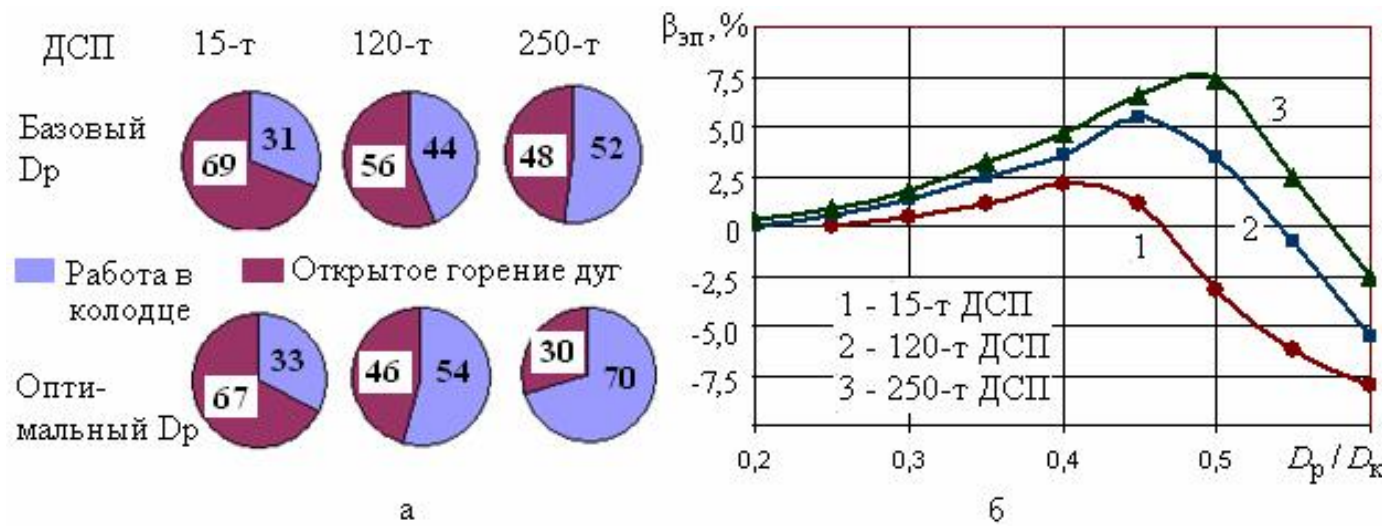

Рисунок 4 - Режимы плавления шихты (а) и относительная экономия электроэнергии $\beta_{\text {эл }}$ в зависимости от $D_{\mathrm{p}} / D_{\text {к }}$ (б) в ДСП различной вместимости

\section{Выводы}

Численное моделирования процесса нагрева и плавления скрапа энергией электрических дуг в условиях ДСП показало, что частный коэффициент энергоэффективности дуг, горящих в индивидуальных колодцах, проплавляемых в шихте, составляет 0,94, снижается до 0,70 при формировании общего колодца и до 0,55 и ниже при открытом горении дуг. Для 120-т ДСП, работающей по интенсивной технологии с загрузкой скрапа бадьями, увеличение диаметра распада электродов $D_{\mathrm{p}}$ до 0,46 диаметра каркаса по панелям $D_{\text {к, }}$ в сравнении с традиционным соотношением $D_{\mathrm{p}} \simeq 0,2 D_{\text {к }}$, ведет к росту относительной продолжительности наиболее энергоэффективной стадии периода плавления - в колодцах на $16 \%$ и снижению продолжительности периода плавления на $4 \%$. Оптимизация $D_{\mathrm{p}}$ позволяет уменьшить удельный расход электроэнергии в крупных (120-250 т) ДСП на 5-6\%, а в печах малой вместимости (12-15 т) на 2-3\%. Оптимальная величина $D_{\mathrm{p}}$ растет от

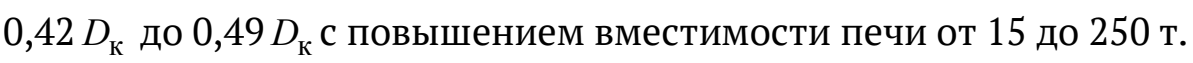

\section{ЛИТЕРАТУРА}

1. Егоров А.В. Расчет мощности и параметров электропечей черной металлургии/ А.В. Егоров. М.: Металлургия, 1990. -280с. 
2. Toulouevski Yu., Zinurov I. Innovation in Electric Arc Furnaces. Scientific Basis for Selection / Berlin (Germany): Springer-Verlag, 2010. - 258 p.

3. Logar V., Dovžan D., Škrjanc I. Modeling and Validation of an Electric Arc Furnace. ISIJ International. 2012, vol. 52, no. 3, p. 402-423.

4. Opitz, F., Treffinger, P. Physics-Based Modeling of Electric Operation, Heat Transfer, and Scrap Melting in an AC Electric Arc Furnace. Metallurgical and Material Transactions. 2016, vol. 47, pp 1489-1503.

5. Stankevich Yu. A., Timoshpol'skii V. I., Pavlyukevich N. V. et al. Mathematical modeling of the heating and melting of the metal charge in an electric arc furnace. Journal of Engineering Physics and Thermophysics. 2009, vol. 82, no. 2, pp. 221-235.

6. Сосонкин O.М., Шишимиров М.В. Анализ факторов, влияющих на угар металла в дуговой сталеплавильной печи / О.М. Сосонкин, М.В. Шишимиров// Электрометаллургия, 2002, № 12, с. 12-15.

7. Timoshenko S.N. Computer modeling bath geometry to improve energy efficiency of electric arc furnace// System technologies, no. 3 (104) - Dnepropetrovsk, 2016, pp.33-39.

\section{REFERENCES}

1. Egorov A.V. Raschet mozhnosti i parametrov electropecej chernoj metallurgii (Metallurgical electric furnaces calculation)/ A.V. Egorov // M.:”Metalluggija”,1990. 280p. (in Russian).

2. Toulouevski Yu., Zinurov I. Innovation in Electric Arc Furnaces. Scientific Basis for Selection / Berlin (Germany): Springer-Verlag, 2010. - 258 p.

3. Logar V., Dovžan D., Škrjanc I. Modeling and Validation of an Electric Arc Furnace. ISIJ International. 2012, vol. 52, no. 3, p. 402-423.

4. Opitz, F., Treffinger, P. Physics-Based Modeling of Electric Operation, Heat Transfer, and Scrap Melting in an AC Electric Arc Furnace. Metallurgical and Material Transactions. 2016, vol. 47, pp 1489-1503.

5. Stankevich Yu. A., Timoshpol'skii V. I., Pavlyukevich N. V. et al. Mathematical modeling of the heating and melting of the metal charge in an electric arc furnace. Journal of Engineering Physics and Thermophysics. 2009, vol. 82, no. 2, pp. 221-235.

6. Sosonkin O.M., Shishimirov M.V. Analiz faktorov, vlijajushirh na ugar metalla v dugovoj staleplavilnoj pechi (The analysis of EAF metal burning factors) / O.M. Sosonkin, M.V. Shishimirov// Electrometallurgija (Electrical metallurgy), 2002, no. 12, pp. 12-15 (in Russian).

7. Timoshenko S.N. Computer modeling bath geometry to improve energy efficiency of electric arc furnace// System technologies, no. 3 (104) - Dnepropetrovsk, 2016, pp.33-39.

Received 22.01.19

\section{ENERGY EFFICIENT SOLUTIONS FOR ELECTRODES PITCH DIAMETER OF ELECTRIC ARC FURNACE}

Analysis of recent research and publications. Modern mathematical models of the scrap heating and melting process in the EAF do not reflect the influence of the electrodes pitch diameter $D_{\mathrm{p}}$ value relative to the diameter of the furnace frame $D_{\mathrm{f}}$ on the EAF energy 
efficiency. Traditional ratio is $D_{\mathrm{p}} \simeq 0.2 D_{\mathrm{f}}$ based on the task of minimization arcs irradiation on the EAF walls refractory lining, which is not actual in modern units with wide using of water cooled panels.

Purpose. To increase the EAF energy efficiency by optimization of $D_{\mathrm{p}}$.

Methodology. Numerical simulations of the arc furnace scrap heating and melting process due to radiation heat exchange, when varying the $D_{\mathrm{p}} / D_{\mathrm{f}}$ ratio, is the subject scope of the paper.

Findings. Energy-efficient solutions for $D_{\mathrm{p}}$ in different capacity EAF, operating by intensive technology with standard bucket charging.

Research implications: Calculation of partial arcs energy efficiency ratios in the individual wells and overall well, which are melted in the charge, shows these values are 0.94 and 0.70 correspondingly. Has been revealed the influence of $D_{\mathrm{p}}$ on the relative duration of closed $\tau_{c a}$ and open $\tau_{o a}$ arcing and, thus, on the total duration of melting period $\tau_{m}$, most energy-intensive in the EAF steelmaking modern technology, as a whole. According to research, in the EAF with an optimal value of $D_{\mathrm{p}} / D_{\mathrm{f}}$ ratio, $\tau_{c a}$ increases by $16 \%, \tau_{o a}$ decreases by $15 \%$ and $\tau_{m}$ decreases by $4 \%$, relative to traditional ratio.

Practical implications. Increasing of the $D_{\mathrm{p}} / D_{\mathrm{f}}$ ratio from traditional 0.2 to (0.42-0.49) $D_{\mathrm{f}}$ improves the energy efficiency of the EAF by changing of the melting mode and leads to energy savings of 5-6\% in large furnaces (120-250 tons) and $2-3 \%$ in small capacity ones (12-15 tons). The optimal value of $D_{\mathrm{p}}$ grows from 0.42 to $0.49 D_{\mathrm{f}}$ with an increase in the EAF capacity from 15 to 250 tons.

Originality. The new concepts of the EAF energy saving working space geometry, based on thermal and technological features of given unit.

Keywords: electric arc steel-smelting furnace, heat exchange, electrodes pitch diameter, energy efficiency.

\section{ЕНЕРГОЕФЕКТИВНІ РІШЕННЯ ДІАМЕТРУ РОЗПАДУ ЕЛЕКТРОДІВ ДУГОВОЇ СТАЛЕПЛАВИЛЬНОЇ ПЕЧІ}

Чисельне моделювання теплообміну в робочому просторі ДСП показало, що для печей 3 інтенсивною технологією збільшення діаметра розпаду електродів 3 традиційного 0,2 до $0,42-0,49$ внутрішнього діаметра каркаса підвищує енергоефективність ДСП за рахунок зростання відносного часу закритого горіння дуг. Зміна режиму плавлення призводить до економії електроенергії на 5-6\% в потужних електропечах (120-250 т) і на 2-3\% в печах малої місткості (12-15 т).

Ключові слова: дугова сталеплавильна піч, теплообмін, діаметр розпаду електродів, енергоефективність.

Тимошенко Сергій Николаевич - к.т.н., с.н.с., ГВУЗ Донецкий национальный технический университет, докторант, г. Покровск.

Timoshenko Sergii - cand. of Sci. (Eng.), Donetsk National Technical University, doctoral, Pokrovsk, Ukraine.

Губинский Михаил Владимирович - д.т.н., професор, ГВУЗ Национальная металлургическая академия Украины, зав. кафедрой, г. Дніпр.

Gubinskij Mikhail - Dr. of Sci. (Eng.), National Metallurgical Academy of Ukraine, professor, Dnipro, Ukraine. 